\title{
Conflit foncier du Goin-Débé à Guiglo en Côte d'Ivoire : Fantôme ethnicisé de la guerre post-électorale ivoirienne de 2010
}

\author{
Djedou Martin Amalaman, \\ Enseignant-Chercheur, \\ Université Peléforo Gon Coulibaly de Korhogo, Côte d'Ivoire
}

Bosson Jean Fernand Mian,

Etudiant, Université de Liège, Belgique

Chercheur, Centre Suisse de Recherches Scientifiques, Abidjan-Côte d'Ivoire

\section{Gnangadjomon Kone,}

Chercheur, Centre Suisse de Recherches Scientifiques, Abidjan-Côte d'Ivoire

Enseignant-Chercheur, Université Alassane Ouattara de Bouaké,

Côte d'Ivoire

Doi:10.19044/esj.2020.v16n10p362 URL:http://dx.doi.org/10.19044/esj.2020.v16n10p362

\section{Résumé}

L'étude s'intéresse à l'épineuse question des conflits fonciers à Guiglo et dans le Goin-Débé, en Côte d'Ivoire.Cette région est le théâtre d'affrontements meurtriers opposants les autochtones Guéré, aux allochtones Agni et Baoulé et allogènes Lobi et Mossi. Ces conflits s'apparentent à de simples crises de cohésion sociale autour du foncier, mais à les saisir comme des choses au sens durkheimien du terme, l'on s'aperçoit qu'ils ne sont rien d'autre que des fantômes de la guerre post-électorale ivoirienne de 2010. À partir d'étude qualitative conduite dans le département de Guiglo entre décembre 2017 et février 2018, l'article a montré que les conflits fonciers dans cette partie du pays, ne sont qu'une réapparition de la guerre post-électorale de 2010. Les causes et caractéristiques de ces conflits ainsi que leurs formes et manifestations, achèvent de convaincre que la guerre post-electorale de 2010, se réinvente en conflits fonciers à Guiglo.

Mots clés : Conflit foncier, ethnicisation, Guiglo, guerre post-électorale, Côte d'Ivoire 


\title{
Land conflicts of Goin Débé in Guiglo in Côte d'Ivoire: ethnicized ghost of the 2010 Ivorian post-electoral war
}

\author{
Djedou Martin Amalaman, \\ Enseignant-Chercheur, \\ Université Peléforo Gon Coulibaly de Korhogo, Côte d'Ivoire \\ Bosson Jean Fernand Mian, \\ Etudiant, Université de Liège, Belgique \\ Chercheur, Centre Suisse de Recherches Scientifiques, Abidjan-Côte d'Ivoire
}

Gnangadjomon Kone,

Chercheur, Centre Suisse de Recherches Scientifiques, Abidjan-Côte d'Ivoire

Enseignant-Chercheur, Université Alassane Ouattara de Bouaké,

Côte d'Ivoire

\begin{abstract}
The study focuses on the thorny issue of land conflicts in Guiglo and Goin-Débé, Côte d'Ivoire. This region is the scene of deadly clashes between the indigenous Guéré, the non-indigenous Agni and Baoulé and the nonindigenous Lobi and Mossi. These conflicts resemble simple crises of social cohesion around the land issue, but if we grasp them as things in the Durkheimian sense of the term, we realize that they are nothing more than ghosts of the post-electoral war in Côte d'Ivoire in 2010. Based on a qualitative study conducted in the department of Guiglo between December 2017 and February 2018, the article showed that land conflicts in this part of the country are nothing more than a reappearance of the 2010 post-election war. The causes and characteristics of these conflicts, as well as their forms and manifestations, complete to convince that the post-electoral war of 2010 is being reinvented as land conflicts in Guiglo.
\end{abstract}

Keywords: Land conflict, ethnicisation, Guiglo, post-electoral war, Côte d'Ivoire

\section{Introduction}

Les conflits fonciers observables ces derniers temps (depuis Novembre, 2017) à Guiglo témoignent en grands partie de la nouvelle typologie que prennent les conflits fonciers dans l'Ouest de la Côte d'Ivoire. 
Cette zone à forte potentialité agricole reflète en grande partie la politique post-indépendante de la Côte d'Ivoire, faisant de l'agriculture le moteur de son économie. De par son attractivité, l'ouest ivoirien subit aussitôt des flux migratoires internes et externes venants des quatre coins du pays. Selon Toh et Koffi, $(2015,1)$, cette dynamique migratoire conflictuelle, en direction de l'ouest Ivoirien, devenu depuis quelques années la nouvelle boucle du cacao, résulte d'une part de la raréfaction des terres arables dans les zones du SudOuest (San-Pedro, Soubré) anciennement boucle du cacao. D'autre part, la crise poste électorale de 2010 et ses effets collatéraux ont favorisé une nouvelle remontée des flux migratoires (déplacement de populations en interne et en externe) dont la descente (le retour des populations) a restructuré considérablement l'arène foncière dans cette tranche ouest du pays précisément à Guiglo. Désormais l'on assiste à des tensions foncières liées aux patrimoines forestiers publics dont l'enjeu majeur est sa conquête et son exploitation. Il s'agit de la forêt classée du "Goin Débé". Ce conflit foncier se caractérise par une triple-réalité socio spatiale car sa forme de manifestation s'opère non seulement dans l'environnement du classé (Goin Débé), dans l'environnement du non classé (espaces agricoles des villages) et dans l'environnement immédiat (espace villageois et urbain). Pour une meilleure approche de cette question foncière, les résultats de l'article se sont articulés autour des séquences suivantes : causes et caractéristiques du conflit du Goin Débé, manifestations et effets collatéraux du conflit, de l'inaction de l'État au sentiment de laxisme et de complicité dans l'accaparement des terres chez les populations "Guéré" de Guiglo.

\section{Methodologie}

À partir d'étude qualitative conduite à Guiglo en décembre, 2017 et février 2018 auprès des principaux acteurs impliqués dans les différents conflits fonciers à Guiglo et surtout dans celui du «Goin-Débé », nous sommes parvenu à la conclusion que le conflit du «Goin-Débé » est le fantôme de la guerre post-électorale ivoirienne de 2010, selon les acteurs interviewés. L'étude a été conduite sous la base d'entretiens sémi-structurés et d'observation participante, au total vingt-cinq (25) personnes ont été inérrogées sous la base de dix (10) entretiens individuels et quatre (4) entretiens de groupe réalisés à Guiglo : Mona, Domobly, Kahadé et dans le Goin-Débé. Les populations cibles choisies par les techniques de choix raisonné et de boule de neige, étaient respectivement: les autorités administratives et coutumières, des agents des eaux et forêts de Guiglo, les populations (jeunes, femmes et chefferies autochtones-Guéré, allochtonesBaoulé-Agni et allogènes Lobi-Mossi), des localités de Mona, Kahadé et du Goin-Débé, ayant vécu fortement l'expérience tragique du conflit. La collecte des données de terrain s'est arrêtée en référence au principe de saturation de 
Bertaux, (1981). Les données ont été fidèlement retranscrites et soumises à une analyse du conténu grace à un traitement méthodique (analyse transversale et comparative par triangulation) à l'aide de fiches de synthèse pour en ressortir les unités de sens et de significations du discours des enquêtés.

\section{Resultats}

Les résultats de cette étude sont présentés sous la base de l'analyse des causes et caractéristiques du conflit du Goin Débé, ses formes et manifestations, ainsi que les jeux d'acteurs des parties en conflit. Ils (ces résultats) achèvent de convaincre avec la thèse selon laquelle, la guerre postélectorale ivoirienne de 2010, n'est pas loin de se transporter et de se rééditer à Guiglo, sous le registre des conflits fonciers.

\section{Causes et Manifestations du conflit du Goin Débé \\ $>$ Causes des conflits}

Le Goin Débé est un patrimoine forestier déclaré classé par l'État Ivoirien depuis 1975. Selon le (RGPH, 2014), le Goin Débé s'étend sur une superficie totale de 133.170 ha et est situé à $100 \mathrm{~km}$ de Guiglo précisément sur l'axe Guiglo Bloléquin. Cette forêt fait partie des 233 forêts classées appartenant au domaine forestier permanent privé de l'État. Couvrant une superficie totale de 4196000 ha (ses forêts classées) sont gérées depuis 1992 par la SODEFOR (société de développement des forêts), gestionnaire unique employant à ce jour 904 agents. Sa mission est de participer à l'élaboration et à la mise en œuvre de la politique du gouvernement en matière d'enrichissement du patrimoine forestier national, de développement et de renforcement de la production forestière et de sauvegarde des zones forestières (Plan d'investissement forestier de la Côte d'Ivoire, 2016). 
Carte 1 : Géolocalisation de la forêt classée du Goin Débé

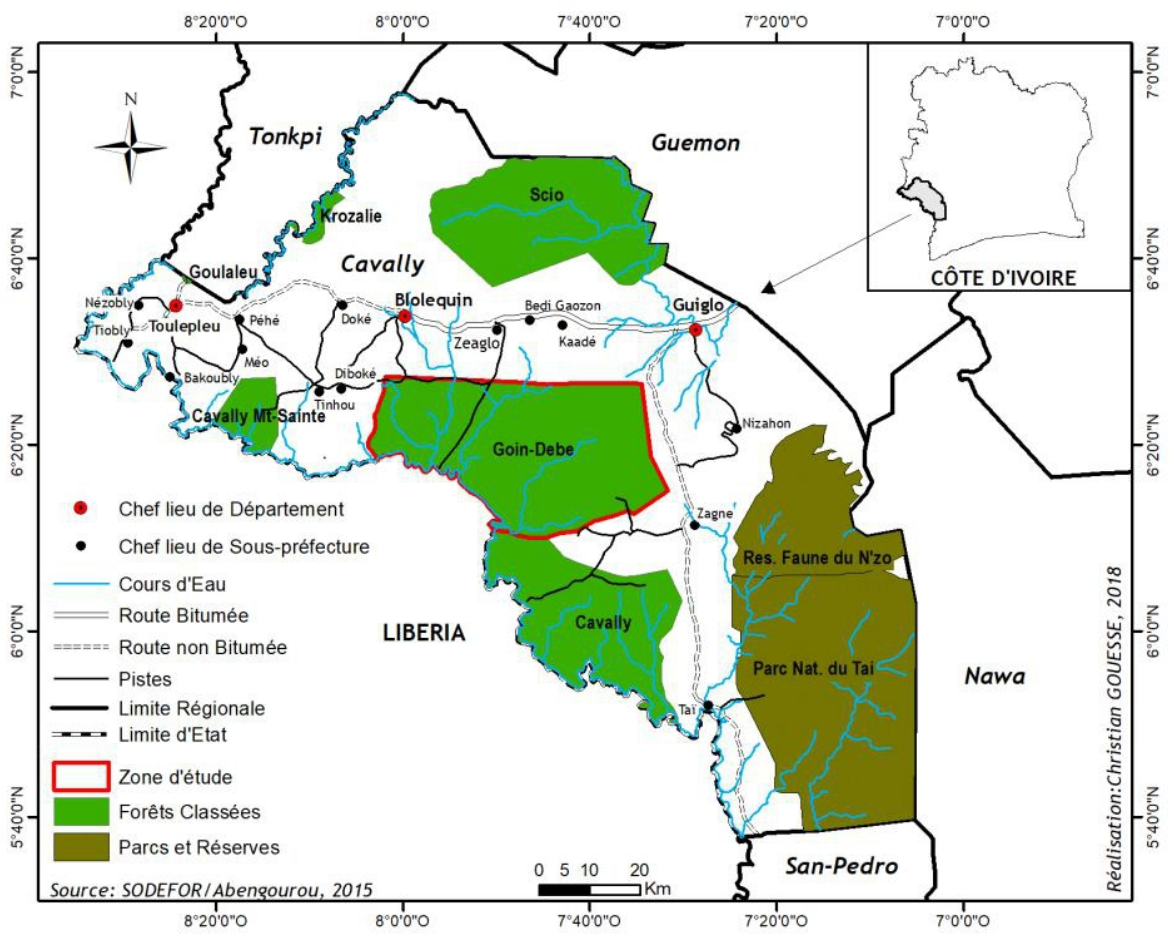

\section{La forêt du Goin Débé : du classée au déclassé}

Les données recueillies ont montré que le Goin Débé faisait objet d'exploitation et d'infiltration depuis la crise post-électorale de 2010, par des allogènes Burkinabé conduits par un dénommé chef Salam, occupant un périmètre d'exploitation de 9000 ha sur les 31000 ha du Goin Débé faisant objet d'exploitations agricoles (selon un chef allochtone baoulé de Guiglo). Ensuite, une seconde occupation illégale et forcée fut constatée par des jeunes autochtones "Wê" après que l'information soit remontée jusqu'à leurs leaders hiérarchiques (chef du village, président des jeunes). Il s'agit d'allochtones Baoulé occupant et exploitant eux aussi depuis très longtemps (bien avant même la crise de 2010) les 22000 ha de la superficie restante du Goin Débé sur la superficie totale faisant objet d'exploitation agricole et même celle-ci (les 9000 ha) occupée par les allogènes Burkinabé car les premiers auraient réussi à chasser les seconds suite à des hostilités relatives à la proportion d'occupation dans le Goin Débé. Cependant, le peuple Wê, riverain de cette forêt avant même qu'elle ne soit déclarée classée est longtemps resté en marge de celle-ci, ce qu'attestent ces propos d'une autorité coutumière de Guiglo : « depuis 1975 que cette forêt est déclarée classée, aucun Guéré n'y met les pieds». Cette absence où mise à l'écart des Autochtones Guéré dans le Goin Débé s'inscrit dans une certaine mesure dans l'idée de citoyenneté et de 
préservation ou protection des patrimoines forestiers ivoiriens classés à travers le respect scrupuleux de ses clauses.

\section{Le conflit foncier du Goin Débé : une conséquence de la «cupidité foncière » des Autochtones Guéré ?}

Bien avant la crise de 2010, certains allochtones baoulés et allogènes burkinabé mossi et lobi qui arrivaient à Guiglo et qui sollicitaient des lopins de terre pour l'activité agricole étaient installés par les Autochtones Guéré dans le Goin Débé à l'insu des agents de la Sodéfor et moyennant de l'argent. C'est ce qu'attestent ces propos d'un chef allochtone baoulé : « en 2008, quand je suis arrivé on m'a dit que ce n'était pas classé et que c'est l'État qui a déclassé ça j'ai même eu 11 ha et c'est après je me suis rendu compte que c'était classé. On entendait dire souvent des Guéré, je vais au classé voir mes travailleurs et le Goin Débé est actuellement dominé par les Burkinabé Mossis et Lobi qui atteignent des millions, on ne sait pas si l'État existe encore en Côte d'Ivoire ». En effet, cette infiltration du Goin Débé occasionnée également par certains Autochtones Guéré dans le temps pour des logiques économiques (les uns se contentaient seulement d'installer les autres sans même y prendre part, sans même songer aux conséquences) n'a fait que constituer un levain dans l'éclatement du conflit foncier du Goin Débé car, ces occupants ayant acquis un certain nombre d'avantages socio-économique dans le Goin Débé au fil des années sont loin de se dessaisir.

\section{Du désaccord et de la frustration à l'idée de conflit autour du Goin Débé}

Le processus de revendication entamé par les jeunes Autochtones Wê, fut caractérisé d'abord par l'ordre de vérification des occupants de la forêt du Goin Débé donné par leurs chefs respectifs. Au cours de cette vérification, les Guéré auraient subi des violences occasionnant la mort, la blessure et la disparition de certains d'entre eux après la découverte de toute une organisation sociale des allochtones Baoulés autour du Goin Débé (existence d'écoles, de marchés, d'église...). « Nos enfants étaient partis les mains nues, les autorités de la SODEFOR ont fait comme s'ils n'avaient rien vu après ce qui s'est passé là-bas », dixit un leader autochtone. On pourrait comprendre que les Autochtones éprouvaient le sentiment d'une banalisation et d'une sorte de négligence de la part des autorités administratives locales sur la situation qui leur avait été rapportée. Or, cette interprétation serait en réalité développée par ces Autochtones Guéré, à la suite d'un échec de compromis entre eux et les Allochtones Baoulés. En effet, les Guéré demandaient aux Baoulé de leur céder le périmètre d'occupation du Goin Débé qu'ils auraient arraché de force de la main des allogènes Burkinabé. Le silence dans la prise de décision des autorités administratives locales conjugué au refus de cession des allochtones Baoulé fut un facteur essentiel d'alimentation et d'interprétation de tous 
genres de la part de ces indigènes, qui n'ont plus qu'à trouver leurs propres satisfactions dans un élan conflictuel. Car selon eux : "le propriétaire ne peut pas être en dehors de sa propriété pendant que d'autres jouissent ». À travers ces propos on comprend que dans l'imaginaire populaire autochtone, l'État a cessé d'être le second propriétaire du Goin Débé à partir du moment où il y a eu des infiltré occupant illégalement cette forêt, il faut donc une revendication de ce droit foncier par le conflit.

\section{Manifestation du conflit du Goin Débé : caractéristiques et pluralisme des acteurs en conflit}

Les hostilités engagées entre les Baoulés et les Burkinabé dans le Goin Débé étaient une question de leadership foncier relatif à la proportion d'occupation des terres, à l'issue desquelles les allogènes Burkinabé furent chassés par les allochtones Baoulés.

\section{L'entrée en conflit des Lobi (enjeu économique du conflit)}

À la suite du refus des Allochtones Baoulés de céder les terres arrachées de la main des allogènes Burkinabé, aux autochtones Guéré, ces derniers vont engager un système de récupération forcé des terres occupées par les Baoulés. Cette action conflictuelle menée par les Autochtones est soutenue par l'idée que « on ne peut pas être au bord de la rivière et puis avoir soif ». Cette affirmation traduit un enjeu purement économique, car dans l'entendement des Guéré, eux-même les propriétaires terriens d'un point de vue coutumier, ne profitent pas de ce patrimoine pendant que les allogènes et allochtones s'en réjouissent, il faut donc changer la situation. Ainsi, la violence du conflit dans le "Goin Débé » oblige les Baoulés à solliciter l'aide des «Dozo » (Lobi) sous proposition qu'après l'évincement des Guéré ils seront dorénavant leur tuteur foncier. Vu l'intérêt, les Lobi entrent en conflit. Mais cette entrée des Lobi serait en réalité soutenue par le fait que lors des tensions entre Guéré et Baoulé certains de leurs parents ont été atteints physiquement. Le sentiment de vengeance conjugué à l'enjeu économique du conflit ne laisse guère indifférent les Lobi.

\section{De la politisation du conflit foncier du Goin Débé}

L'une des variables majeures de la dimension politique du conflit foncier du «Goin Débé » reste la perception des Autochtones de la présence d'acteurs latents ou indirects dans l'occupation (par acteurs interposés) des terres du Goin Débé. En effet, cette représentation autochtone de l'occupation du Goin Débé se perçoit sur l'échiquier politique dans la mesure où la localité même de Guiglo a une population à forte dominance FPI (Front populaire Ivoirien) ex Parti politique au pouvoir en Côte d'Ivoire. Ainsi, l'on va assister à une récupération politique du conflit foncier du Goin Débé dès lors que les 
Autochtones Guéré découvrent que les occupants latents (réels) de cette forêt dite classée sont certains représentants politiques de leur principal parti d'opposition qu'est le RHDP (rassemblement des "Houphouëtistes" pour la démocratie et la paix). Aussitôt, un système de récupération est mis en place par un leader autochtone FPI. Il s'agit de l'alliance des jeunes Wê dont la stratégie consiste en une insoumission aux autorités administratives locales en qui les Autochtones voient une complicité avec le pouvoir en place comme rapportée ci-dessous : "quand les autorités invitent les jeunes Guéré à un dialogue, un leader alimente les consciences parce que, être Guéré c'est être propriétaire terrien et non simple récolteur de cacao ». On comprend de loin que, ces autochtones Guéré ont en réalité du mal à accepter le fait qu'ils soient en marge des terres du Goin Débé au moment où des Allochtones et allogènes en exploitent tranquillement sous couverture de certains de leurs dirigeants politiques du parti au pouvoir. Toutefois, cette frustration relative des Guéré va animer davantage les instincts de conflictualité à partir du moment où elle traduit le sentiment d'injustice dans la gestion du conflit ; «ce sont les Lobi seulement qui sont arrêtés dans toutes les prisons, aucun Baoulé n'est arrêté », affirma un fils de Guiglo. Ces propos qui expriment l'idée de discrimination et d'impartialité complexifient la résolution du problème en question. Par ailleurs, ils constituent une preuve afférente de la véracité de la politisation du conflit foncier du Goin Débé que fustigent les Autochtones Wê.

\section{Des manifestations et effets collatéraux du conflit du «Goin-Debe »}

La frustration vécue par les autochtones Guéré de Guiglo quant à l'occupation illégale des Allochtones (Baoulés et Lobi) et allogènes (Burkinabé) des terres du Goin Débé va susciter la naissance de l'alliance des jeunes wê, crée par "Oulaï Iber" (leader FPI de Guiglo). Elle fut instituée comme un système de revendication et de récupération du Goin Débé et comprend les jeunes wê des localités de Guiglo, Bloléquin, et Taï. En effet, ce système mis en place est soutenu par la logique d'une lutte visant la recherche de conditions de subsistances meilleures des autochtones qui se sentent dépossédés de leur pouvoir économique agricole. À cet effet, pour un responsable des jeunes d'un village de Guiglo : «il y aura toujours une revendication si nos frères mangent et que nous on ne mange pas, et même si nos luttes n'aboutissent pas tôt ou tard nos fils continueront; on nous parle de cohésion sociale pendant que nous mourons de faim, nous demandons un signal fort et équilibré au gouvernement sinon on peut rentrer au Libéria chez. nos frères et les mobiliser à venir faire la guerre ». À travers ces propos, on comprend non seulement l'amertume qu'éprouvent les autochtones Wê face à cette situation mais aussi combien de fois ceux-ci aspirent à une transformation et amélioration durables de leurs conditions de vie socioéconomiques garant d'un climat social de paix durable. Dans l'entendement 
de ces Autochtones Guéré, leur existence sociale et celle de leurs petits-enfants dépendent des terres du Goin Débé. Pour cela, la stratégie de la lutte consiste à préparer aussitôt les jeunes consciences à l'idée de conflit mais aussi à solliciter dans le pire des cas des soutiens extérieurs pour défendre leurs causes si des solutions équitables, durables et satisfaisantes pour tous ne sont pas trouvées. Dans un tel contexte on ne pourrait qu'assister à une perpétuation et à une internationalisation (entrée de nouveaux acteurs) du conflit foncier du Goin Débé. Il faut donc contenir immédiatement le problème par des mesures durables et impartiales.

\section{La légitimation de leurs actions par le décès de leur frère}

Les hostilités entre Baoulés, Lobi et Guéré survenues dans le Goin Débé ont transformé l'environnement de Guiglo en un champ de violence ciblée et caractérisée par un système de chasse à l'homme.

L'assassinat du président des jeunes de Béoua par des inconnus et ses effets collatéraux

Un Autochtone Guéré du village de Béoua à $16 \mathrm{~km}$ de Guiglo fut tué par des inconnus. Mais la population attribue cet acte criminel à un Lobi. La victime (sur l'image) se nomme Danéaho Évariste, et est le président des jeunes du village de Béoua.

Photo 1. Des images du conflit foncier du Goin Débé (Enquête décembre, 2017)

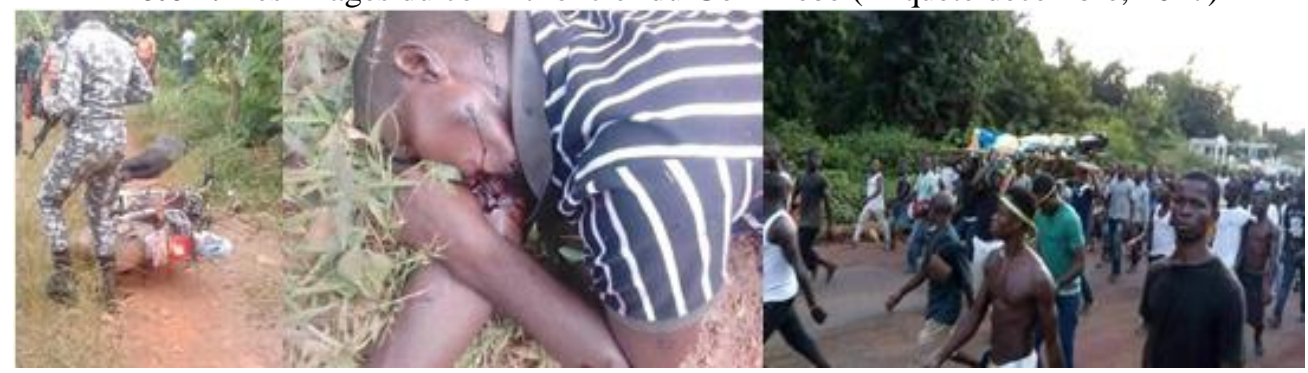

L'information va remonter jusqu'au préfet de Guiglo, qui selon les informateurs Guéré l'a banalisé sans aucune justification satisfaisante, ne leur laissant aucun autre choix que le recours aux actes de vandalismes. D'où cette affirmation d'un responsable des jeunes d'un village de Guiglo : "après avoir informé le préfet il a pensé que c'était de la rumeur, et on a transporté le corps du défunt de Bewa à $16 \mathrm{~km}$ de Guiglo jusqu'à la préfecture ce qui a entrainé la mise en fumée de la préfecture, le pillage du domicile du préfet, et de la gendarmerie et le pillage et l'incendie du domicile du chef de la communauté des baoulé»

Concernant les conséquences : 6 Burkinabé tués à Zéaglo ; 2 Wê tués (1 dans la forêt classée et le président des jeunes a Beoua très loin de forêt classée); un pasteur et son fidèle portés disparus à Beoua; 2 wê portés disparus. 
Comme autres conséquences, nous avons : l'incendie de la résidence du chef de la communauté Akan de Guiglo ( Mr Méa), et le saccage des locaux de la préfecture et de la résidence du Préfet, (Mr Méssamba, un préfet militaire et ex-chef de guerre de la rébellion).

Photo 2 : Des images des effets collatéraux du soulèvement des jeunes Wê.

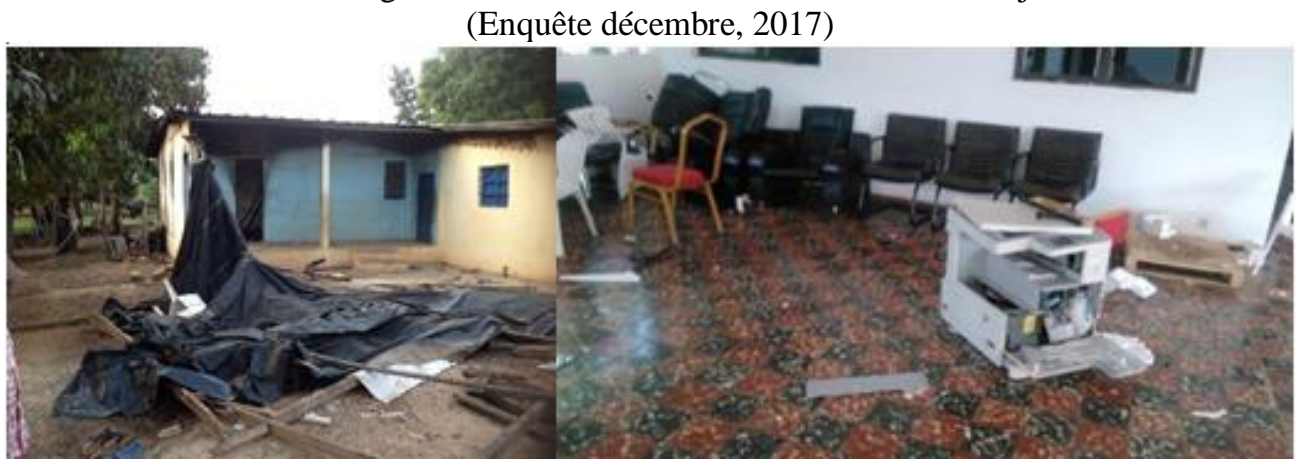

On pourrait tirer de ces propos que cette attitude des Autochtones aussi déplorable et violente qu'elle soit, fut alimentée par des logiques d'expression d'un ressentiment politique de gouvernance longtemps digéré par ceux-ci. En effet, nos données ont révélé que le préfet de Guiglo serait un ancien militaire ex comatant de la rébellion armée de Cote d'Ivoire. Donc transporter le corps de leur frère tué en marchant à plus de $16 \mathrm{~km}$ et le poser devant le préfet, est une symbolique profonde qui atteste non seulement de la complicité du préfet et de ses collaborateurs administratifs locaux (CB de la gendarmerie) dans la mort de leur frère mais aussi du fait qu'ils en avaient gros sur le cœur.Ainsi, la frustration ressentie par les Autochtones Guéré à l'idée d'être sous l'autorité d'un membre de leur principal parti politique d'opposition, conjuguée à son attitude de favoritisme vis-à-vis des allochtones Baoulés (principaux adversaires du conflit foncier du Goin Débé) fut en réalité un facteur significatif et justificatif de leurs actes posés .

\section{Du sentiment d'insécurité à la réclamation du départ de certains administrateurs locaux}

Les conflits fonciers opposant les autochtones Guéré aux Allochtones Baoulés ont suscité un véritable climat d'insécurité sociale à Guiglo. Et l'une des conséquences majeures fut la réclamation par les Autochtones du départ de certaines autorités administratives (Commandant de la brigade de gendarmerie, le responsable de la SODEFOR, le préfet de Guiglo) car dans l'entendement des Guéré, ces autorités seraient en complicité avec les Baoulés, ce qui expliquait la lenteur et la négligence dans la prise de décision sur les faits qui leur étaient rapportés et par ricochet la persistance du problème. Par ailleurs, ce sentiment d'insécurité s'exprimait par une attitude 
de méfiance entre les différents acteurs en conflits (Guéré, Lobi, Burkinabé et Baoulés) qui se regardent de plus en plus en chien de faïence. Il nous a été rapporté que : "une scène de violence a éclaté entre deux Lobi, ou l'un a découpé l'autre à la machette croyant que c'était un Guéré ». Ce comportement de violence s'inscrit dans une sorte de représailles entre Lobi et Guéré dont la justification se trouve dans le fait que les premiers accusent les seconds d'avoir causé du tort à certains de leurs parents lors des tensions dans le Goin Débé. Ainsi, cette violence entre ces catégories d'acteurs, qui au départ se circonscrivait autour du Goin Débé va se déporter à petit feu à Guiglo et dans les villages environnants en se muant en un véritable conflit intercommunautaire dans lequel les autochtones Guéré se sentent davantage livrer à eux-mêmes. Comme l'atteste ces propos d'autochtones Guéré : "Après négociation nous avons accepté leur retour mais eux allogènes et allochtones nous empêchent d'aller au champ, pour aller cueillir son cacao mon grand frère est obligé de se faire accompagner par les militaires ». Il s'agit là d'une volonté de changement de situation exprimée par les Guéré qui animés par un sentiment de peur profond pensent être sans aucun soutien face à des allogènes et allochtones de plus en plus alliés. Or dans une telle situation, il est probable qu'on ait recours à d'autres forces extérieures. Ce qui peut constituer un facteur de perpétuation de ces conflits si des mesures idoines, équitables et immédiates ne sont pas trouvées.

\section{De l'inaction de l'État au sentiment de laxisme et de complicité dans l'accaparement des terres chez les populations Guéré de Guiglo}

Le sentiment de frustration qui anime les Autochtones Guéré de Guiglo suite à l'inaction, au sentiment de laxisme et de complicité qu'ils ressentent vis-à-vis de l'État dans l'accaparement de leur terre trouve préalablement sa racine dans la lenteur des administrateurs locaux dans la prise de décisions équitables et durables quant au conflit du Goin Débé d'où l'affirmation de ce chef autochtone: " l'État est responsable parce que ça été déclassé par l'État et occupé illégalement par les allogènes et les allochtones, classé en faveur des Autochtones et déclassé en faveur des autres, on ne peut pas être là et puis voir les étrangers occuper notre patrimoine ». Il ressort de ces propos que dans l'entendement des Autochtones, l'État instrumentalise son statut de garant du patrimoine foncier ivoirien pour légitimer d'une manière à une autre la présence des allogènes et des allochtones dans le Goin Débé. Offrant ainsi, aux allochtones la possibilité d'être doublement propriétaire foncier sur leur propre territoire. Cette perception des Autochtones semble être éclairée implicitement par ces propos de certains responsables administratifs de Guiglo : «selon nos données de rapport, le vrai problème c'est la faiblesse de l'État, il y a eu une définition des espaces entièrement protégés après le détachement de la SODEFOR et des eaux et forêts, normalement tous ces 
patrimoines ne doivent plus faire objet de conflits fonciers parce que ça fait partir du patrimoine de l'État, une forêt classée c'est une forêt classée ». Ensuite ce sentiment de complicité et de laxisme nourri par les Autochtones à l'égard de l'État prend ici une autre valeur de manque de solidarité et de privation de l'État. On le remarque à travers ces propos recueillis : «la situation n'a pas encore été réglée, pas de compensation, ou d'indemnisation et on nous parle de cohésion sociale, dans le mont Peko, les plantations ont été détruites au moment où le Goin Débé est resté intact, ou bien on détruit la forêt du Goin Débé, incendié Goin Débé et reboiser ». On pourrait d'une part comprendre de ces propos qu'il y a un manque d'actions réelles de la part de l'État qui soit susceptible d'apporter un véritable soutien et réconfort moral ou physique visant en l'apaisement des Autochtones qui se sentent de plus en plus victimes et lésés dans ce conflit. D'autre part la protection ou le fort rattachement de l'Etat au Goin Débé ainsi qu'aux occupants actuels suscite davantage dans l'esprit des autochtones ce sentiment de complicité de l'État, qui refuse de rechercher d'éventuelles solutions dans la destruction du Goin Débé. Ceux-ci considèrent cette inaction étatique comme un moyen de leur exclusion sociale des terres du Goin Débé.

\section{Discusion}

Ce présent article a porté sur le conflit foncier du « Goin Débé », une nouvelle typologie de conflit officiellement reconnue dans la sphère du foncier en Côte d'Ivoire en raison de son impact socio-politique surtout dans l'ouest $\mathrm{du}$ pays. La cause principale de l'éclatement du conflit foncier du «Goin Débé » peut se résumer aux macros-facteur qu'est l'infiltration clandestine du "Goin Débé » dont les corolaires sont ( la fragilisation du contexte sociopolitique du pays de 2000 à 2011 , l'ambiguité des politiques forestières conjuguée au laxisme, à la complicité et à l'avidité d'acteurs politiques et de leurs avals ainsi que les manœuvres économiques d' autochtones ).Notre étude rejoint sur cet aspect ceux d'Amani, $(2011,143)$ et Atta et al, $(2017,357)$. Le premier qui a abordé sous un angle un peu plus macro, la question des infiltrations des forêts classées en Côte d'Ivoire, explique que les logiques de ces infiltrations des populations paysannes sont occasionnées par des approximations des politiques forestières ivoiriennes, ainsi identifie-t-il les faiblesses des politiques de gestion à deux niveaux à savoir : l'élasticité de la propriété foncière et son accessibilité illimitée dans le classer. Les seconds quant à eux, ont analysé les situations conflictuelles nées de l'occupation des forêts classées par les populations de Béki et Bossematie dans la région de l'Indénié-Djuablin (Abengourou), ils arrivent à la conclusion que cette infiltration multi-ethnique ( nationaux et non nationaux) dans cette partie Est ivoirienne est moins soutenue par des facteurs économiques que par des logiques de manque d'équité sociale dans l'administration forestière. Toujours 
dans ces mêmes logiques sous-tendant les conflits fonciers, notre travail rejoint celui d'Alladjaba et Tchékote (2018) mais dans un contexte d'analyse différente. À travers leur étude dans la sous-préfecture de Mongo, Région Du Guéra (Tchad), les auteurs ont abordé la question des conflits sous la base de leur impact sur le développement des campagnes, en montrant que la forte dépendance des populations majoritairement paysannes de la ressource terre, comme source de couverture de leurs besoins alimentaires, fait de l'accès à cette ressource la pierre angulaire de cette compétition conflictuelle. Par ailleurs, le conflit foncier de la forêt classée du « Goin Débé a enregistré des conséquences relativement lourdes en matière de pertes en vies humaines, de destruction d'habitations et de migration forcée. Cette dimension surtout violente que requiert le conflit foncier du « Goin Débé » outre les éffets induits achèvent de convaincre que cette recherche se rapproche également et surtout de celle réalisée par: «Kouakou, (2015, 58). Mémoire de Master en Sociologie et Anthropologie : Socio- histoire de la violence criminelle autour du foncier rural dans l'ouest Ivoirien (cas de Fengolo $S / P$ de Duékoué). Université Alassane Ouattara de Bouaké (Côte d'Ivoire) ».

\section{Conclusion}

Au demeurant, il convient de retenir que cette étude sur le conflit foncier du «Goin Débé » a retracé progressivement les causes et les caractéristiques du conflit, les acteurs impliqués et les effets collatéraux induits par ces conflits. Ce débat conflictuel autour du "Goin Débé" dont les principaux belligérants sont les Autochtones Guéré, les allocthones Baoulés et Agni et les allogènes Burkinabé Mossi et Lobi, a révélé à quel point la terre constitue encore aujourd'hui la seule et unique ressource de subsistance en milieu rural Ivoirien. Ainsi, son acquisition mal négociée tant au niveau des communautés rurales et des administrateurs publics est susceptible d'entrainer une perturbation mortifère du climat social. Par ailleurs, l'étude a également permis de comprendre que, les conflits fonciers en Côte d'Ivoire en général et plus particulièrement dans la partie Ouest, prennent une nouvelle dimension. Il s'agit en fait d'une mouvance de ces conflits vers la « sphère du classé », traduisant la question cruciale de la disparition du couvert forestier du pays. Selon les données de notre recherche, cet état de fait est dû en réalité à une situation malheureuse dont la responsabilité incombe en grande partie à cette machine Etatique dont le laxisme, traduit le comportement analogue à celui " d'un homme avec une plaie au genou et qui refuse de la soigner au risque de se faire mal ». Toutefois, les meurtres, la situation d'insécurité induite par ces conflits, ainsi que les logiques socio-économiques et politiques les ayant alimentée, nous conforte à les qualifier de « fantôme de la guerre post-électorale ivoirienne de 2010. 


\section{References:}

1. Acker, V. F and al (2001/4)."Les «maï-maï » et les fonctions de la violence milicienne dans l'est du Congo." Politique africaine 84: 103116.

2. Akindès, F. (2015), (sous la direction de). Côte d'Ivoire: la réinvention de soi dans la violence, série de Livres du CODESRIA.

3. Amani, Y C, (2011). Logiques des infiltrations paysannes dans les forêts classées en Côte d'Ivoire. European Journal of Scientific Research, ISSN, vol 01 PP, 143-152.

4. Atta, J M K, and al, (2017). Déforestation et conflit foncier rural dans les forêts classées de Béki et Bossematie dans l'Est da la Côte d'Ivoire. Éd. Universitaires Européennes ; Editors. Ed, pp 357-387.

5. Babo, A. and Droz Y. (2008/4)."Conflits fonciers. De l'ethnie à la nation ? Rapports interethniques et « ivoirité » dans le sud-ouest de la Côte d'Ivoire." Cahiers d'études africaines 192(p.741-764).

6. Gaouli bi A.P (2012) : «Tutorat et conflits fonciers ruraux dans l'Ouest Ivoirien : cas de Fengolo », In rapports de recherche du CODESRIA. $\mathrm{N}^{\circ} 16$ (chaine UNESCO pour la paix, Université de Cocody, Abidjan, Cote d'Ivoire).

7. Kouakou, M, R. (2015). Mémoire de master en sociologie et Anthropologie : Socio- histoire de la violence criminelle autour du foncier rural dans l'ouest Ivoirien (cas de Fengolo S /P de Duékoué). Université Alassane Ouattara de Bouaké (Côte d'Ivoire).

8. Toh A et Koffi. E. L, (2015). Dynamiques migratoires, enjeux fonciers et conflits fonciers dans un contexte de retour des populations autochtones déplacées dans l'Ouest Ivoirien. Communication atelier sur migration et enjeux fonciers en Côte d'Ivoire ; JAGA 2012-2016. Base. Afrique. Gouvernance.Net.

9. Zongo M, (2010). Migration et conflits fonciers. Fonds documentaire dynamique sur la gouvernance des ressources naturelles de la planète, AGTER. Dossiers fonciers en Afrique de l'ouest, fiche pédagogique. 